Aims(s) The service was launched in 2013 covering a population of 3.5 million. It has significantly improved the care of patients who develop this distressing condition across our region. Education is integral to the role and has raised awareness amongst patients and clinicians of the early signs.

Methods The service provides a single point of contact for all health-care sectors. It offers advice and coordinated care based on evidence-based guidelines and a robust network pathway. Patients are triaged and referred for surgery, radiotherapy, systemic treatment or best supportive care. All patients are offered rehabilitation. Data are collected prospectively for service evaluation.

Results Evaluation has demonstrated significant improvements. In the first four years, 2635 patients were referred. 96\% had an MR scan within 24 hours, out of these, 1133 had confirmed MSCC and 345 had impending cord compression. $16 \%$ of patients were treated with surgery (this is a three-fold increase since before the service) and 64\% with radiotherapy, also within 24 hours.

Conclusion Awareness amongst patients and clinicians has significantly increased resulting in timely diagnosis, treatment and rehabilitation. Earlier diagnosis also has the potential to prevent MSCC in the future. Back pain is managed earlier and patients remain ambulant with improved survival, reducing hospital admissions with overall financial benefits.

\section{0-10 METASTATIC SPINAL CORD COMPRESSION PATIENT SURVIVAL FOLLOWING ESTABLISHMENT OF A COORDINATOR SERVICE}

Lena Richards, Vivek Misra, Claire Shanahan. The Christie NHS Foundation Trust, Manchester, UK

\subsection{6/bmjspcare-2019-HUKNC.10}

Background Clinical trials have shown surgery to be superior to radiotherapy for patients with metastatic spinal cord compression (MSCC). We present our results following the establishment of a centralised MSCC service, the aim of which is to coordinate the pathway of patients with MSCC within 15 hospitals that serve a population of 3.5 million people.

Aims The primary aim of this study was to determine the survival time following treatment for metastatic spinal cord compression. The secondary aim was to compare our results against survival times quoted in the medical literature.

Methods Data was prospectively collected and analysed for all patients referred with confirmed MSCC in 2014 and 2015. Patients were triaged by the oncologist and spinal surgeon, based on prognosis and performance status and categorised into four subtypes: (1) best supportive care (2) systematic anti-cancer therapy (3) spinal surgery and post-operative radiotherapy (4) radiotherapy alone.

Results 1253 patients were referred to the MSCC service, of which 540 (43\%) had confirmed MSCC. 73 patients (14\%) received best supportive care (median survival of 32 days), 24 patients (4\%) had systemic anti-cancer therapy (median survival of 637 days), 89 patients (16\%) had surgery (median survival of 377 days) and 354 patients (66\%) had radiotherapy (median survival of 62 days).
Conclusion Our results of 377 days compare favourably to studies by Patchell and Fehling, which found median survivals of 126 days and 230 days respectively following surgery. Our data strongly supports the use of decompressive spinal surgery with instrumented stabilisation for MSCC in carefully selected patients.

\section{0-11 DEACTIVATION OF IMPLANTABLE CARDIAC DEFIBRILLATORS AT END-OF-LIFE: INTEGRATED WORKING IS ESSENTIAL}

${ }^{1}$ Fiona Hodson, ${ }^{2}$ Julia DeCourcey, ${ }^{2}$ Stefan Karwatoski, ${ }^{2}$ Mohammed Albarjas, 'Joy Ross, ${ }^{1}$ Rob George. ${ }^{1}$ St Christopher's Hospice, London, UK; ${ }^{2}$ King's College Hospital Foundation Trust, London, UK

\subsection{6/bmjspcare-2019-HUKNC.11}

Background The benefits of integrated cardiac and palliative care support for patients with end-stage heart failure (ESHF) is well recognised (Jaarsma, Beattie, Ryder et al., 2009; Hospice UK, 2017). NICE guideline support use of implantable cardiac defibrillators as part of optimal treatment. Guidelines recommend early discussion with 'careful, explicit, shared-decision making' to promote timely deactivation at end-of-life (NICE, 2014; British Heart Foundation, 2007). In practice this presents challenges for both professionals and patient/family, with varying and limited access to deactivation in the community setting.

Methods A case series is presented from a service evaluation of an integrated model of care for ESHF patients. 10/89 patients had implantable cardiac resynchronization therapy defibrillators (CRT-D) and $1 / 89$ an implantable defibrillator (ICD). We present some of the challenges in management of these devices in our palliative cohort.

Results 8/10 devices were deactivated due to acute deterioration of patients; $1 / 10$ patients reported shocks given. Timing of deactivation was arranged according to the patient's clinical condition and wishes, predominately within a month of death. Deactivation occurred in a variety of settings: hospital inpatient (3), hospital outpatient (3), home (1), hospice (1), including visits by electrophysiologists from tertiary centres and temporary use of magnets by clinicians.

Discussions around deactivation of the CRT-D were complex due to previous clinical history and ingrained patient/family perceptions. Discussions were always multiprofessional; one case was taken to the Hospice Clinical Ethics Committee. One patient had the defibrillator function deactivated and was subsequently resuscitated by the Ambulance Service when he went into fast atrial fibrillation.

Family involvement and agreement with decision making was key, as was joint discussions by different professionals across care settings including those who had known the patient longest.

Conclusion These cases illustrate the need for further discussion and protocols regarding the time and place of deactivation of defibrillators at end of life, and also the challenges for professionals of having resuscitation discussions with these patients and importantly their families. 